\title{
15 years of paediatric intracranial abscess formation - the role of ENT
}

Stephen Ball, Fabian van der Velden, Marieke Emonts-le Clerq

Newcastle University \& Newcastle Hospitals, Newcastle upon Tyne NE2 4HH, UK

\section{Aims}

- Investigate the local patient population and management

- Determine the local microbiological profile and antibiotic resistance patterns

- Review antibiotic practices and consider suggestions for empirical treatment

\section{Methods}

- Retrospective, single-centre cohort study

- January 2001 - June 2016

- Patient identification: clinical coding and discharge books

- Data collection: case notes and digital resources

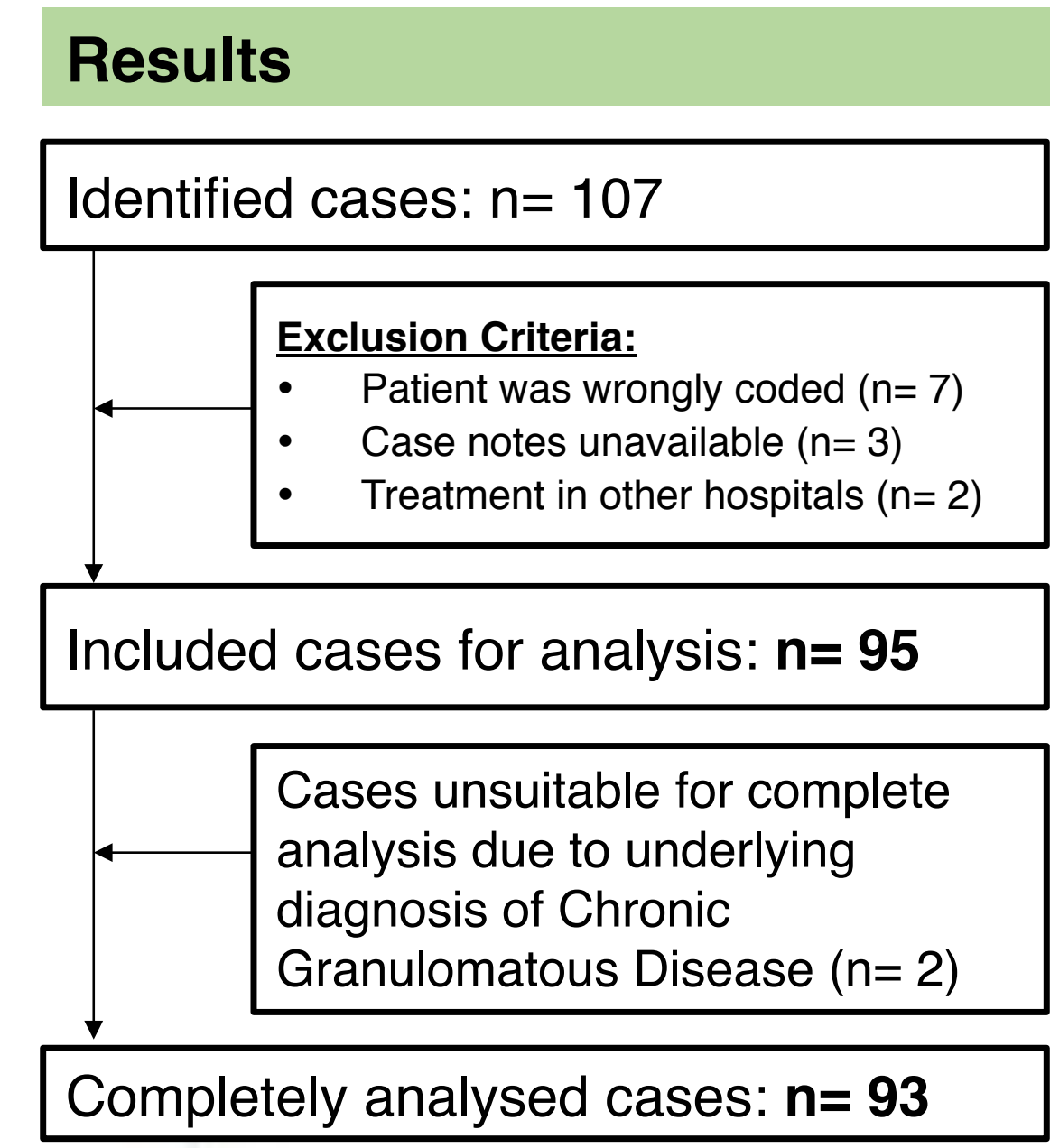

Figure 1: Flowchart of Patient Selection

\section{Erasmus MC}

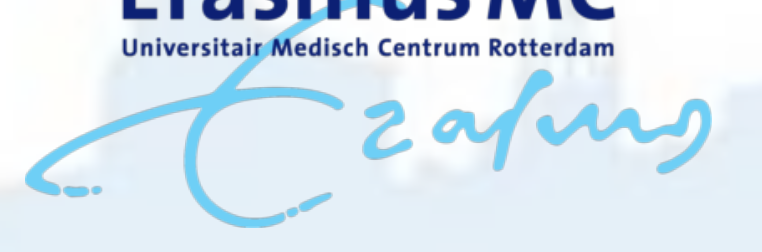

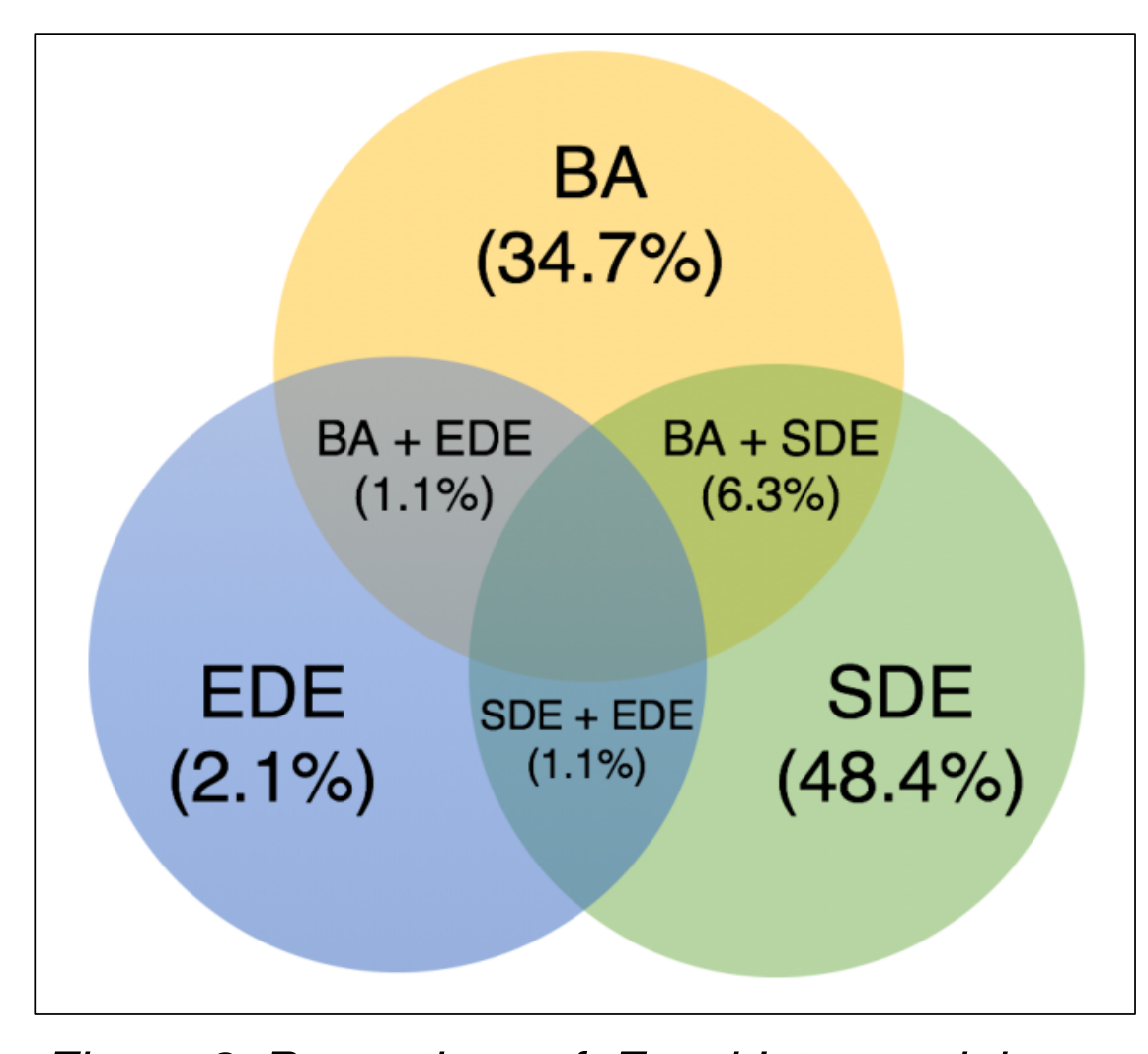

Figure 2: Proportions of Focal Intracranial suppuration; Brain Abscess (BA), Subdural Empyema (SDE), and Extradural Empyema (EDE)

- ENT sources contributed $61.1 \%$ of infections

- Estimated annual incidence: $\mathbf{8 . 7 9}$ per million

- $\mathbf{4 3 . 8 \%}$ of isolates were Streptococci

- Anaerobes not seen in infants less than 1 year

- Neurosurgical interventions in $\mathbf{8 0 . 6 \%}$ $(n=75)$

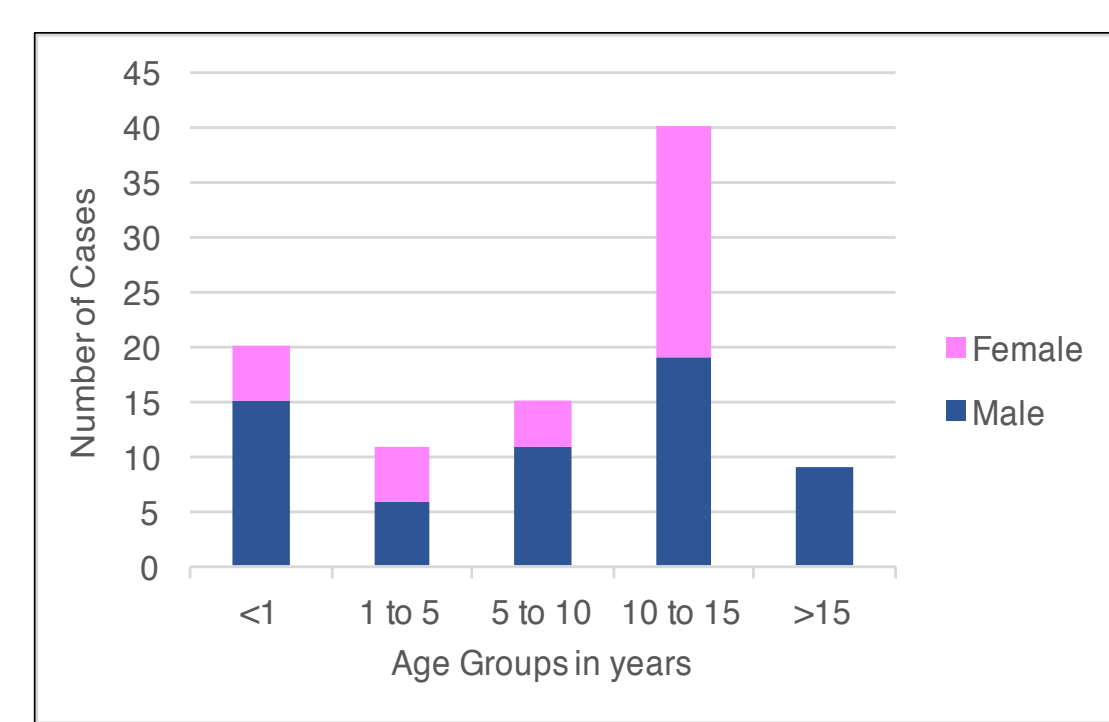

Figure 3: Age Distribution of Focal Intracranial Suppuration by Age Groups

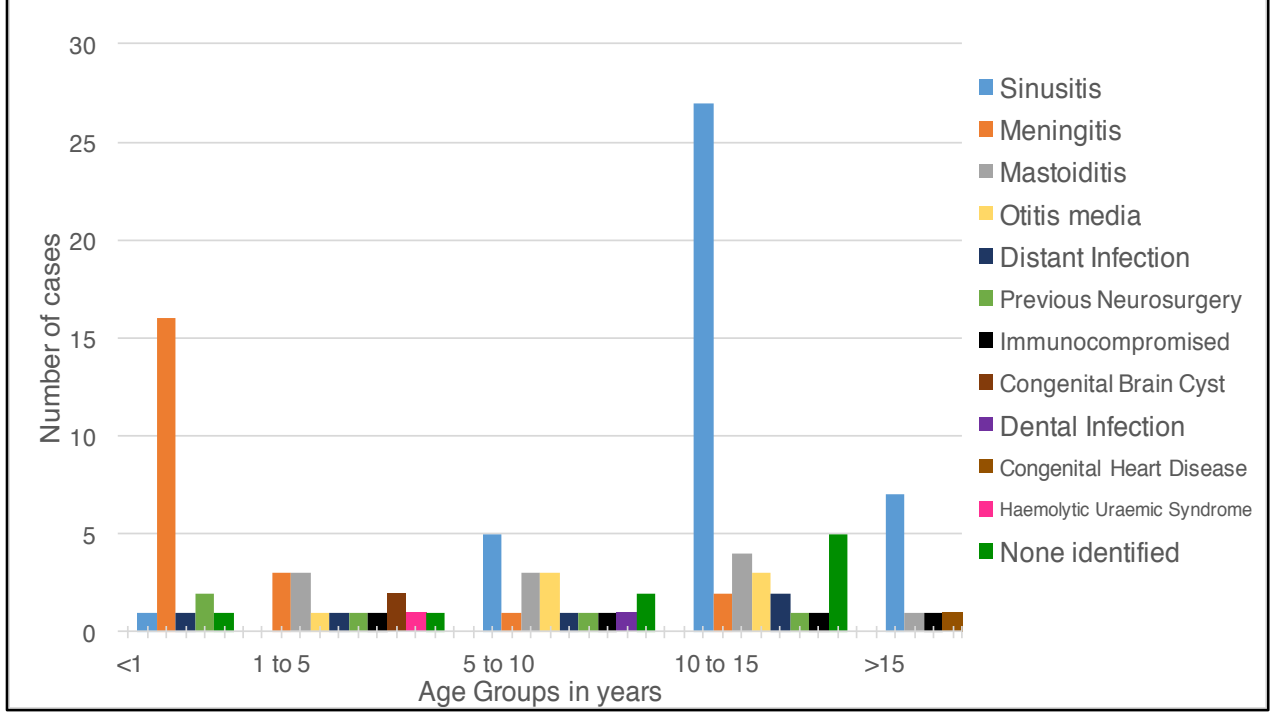

Figure 4: Source of infection by Age Groups

- 31 empirical regimens, 17 different antibiotics given in this cohort.

- Most common regimen: 3rd generation cephalosporin, amoxicillin and metronidazole

- Sufficient antibiotic coverage:

- $95.2 \%$ by empirical regimen

- $90.5 \%$ by $3 \mathrm{rd}$ generation cephalosporin + metronidazole

- $95.2 \%$ by meropenem + linezolid

Total antibiotic treatment duration: 92 days (IQR, 59-119 days)

- Conversion to oral antibiotics: $66.3 \%$

- Mortality 3.2\% $(n=3)$

- Neurological sequelae: $\mathbf{3 8 . 0 \%}$ short-term; 23.9\% long-term

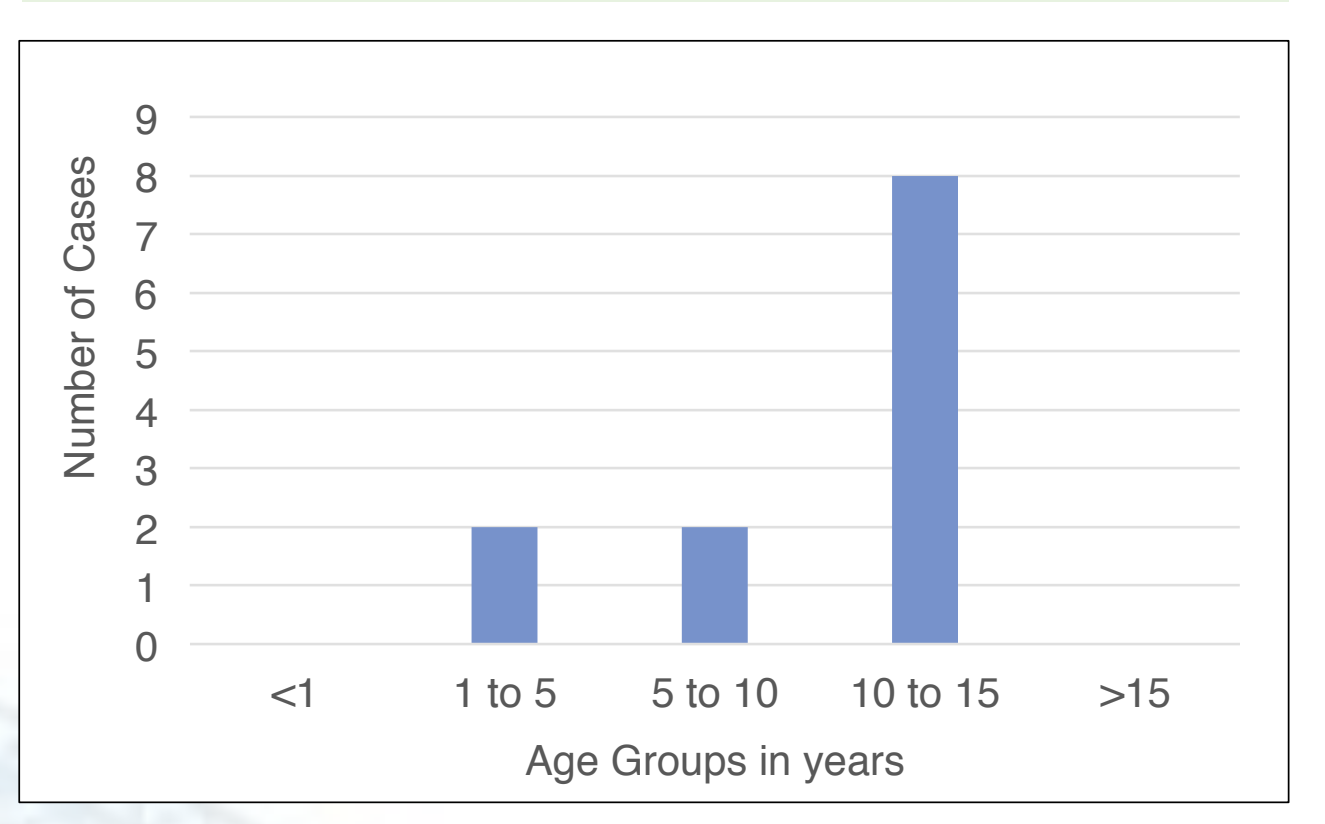

Figure 5: Distribution of patients with positive anaerobic cultures by Age Groups

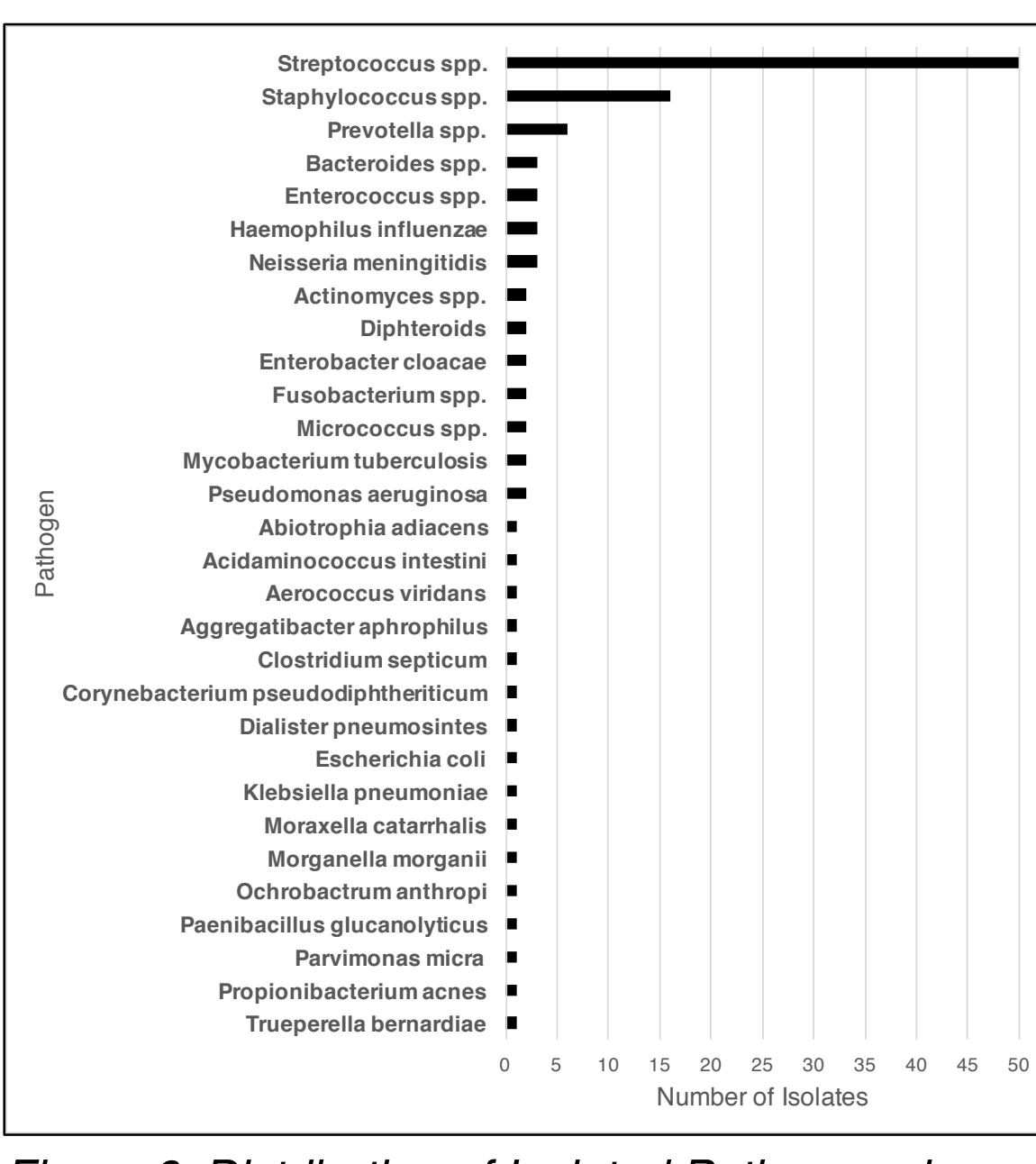

Figure 6: Distribution of Isolated Pathogens in Growth-Positive patients $(n=114)$

\section{Conclusions}

- Focal intracranial suppuration continues to cause significant mortality and morbidity

- ENT infections were the most common source of intracranial suppuration.

- Recommended empirical antibiotic regimen: $3^{\text {rd }}$ generation cephalosporin + metronidazole

- In infants with negative anaerobic cultures:

consider early discontinuation of metronidazole

The Newcastle upon Tyne Hospitals WHS NHS Foundation Trust 